\title{
Lifestyle and nutrition, caloric restriction, mitochondrial health and hormones: Scientific interventions for anti-aging
}

Luis Vitetta'

Bill Anton ${ }^{2}$

'Unit of Health Integration, School of Medicine, University of Queensland, Australia; ${ }^{2}$ Path Lab, Melbourne, Australia
Correspondence: Luis Vitetta Level 2, Centres for Health Research [R Wing], Room 2RS.36.0, Princess Alexandra Hospital, Ipswich Rd, Woolloongabba, Qld 4102, Australia Tel +6I 732402903 Email l.vitetta@uq.edu.au

\begin{abstract}
Aging is a universal process to all life forms. The most current and widely accepted definition for aging in humans is that there is a progressive loss of function and energy production that is accompanied by decreasing fertility and increasing mortality with advancing age. The most obvious and commonly recognised consequence of aging and energy decline is a decrease in skeletal muscle function which affects every aspect of human life from the ability to play games, walk and run to chew, swallow and digest food. There is hence a recognised overall decline of an individuals' fitness for the environment that they occupy. In Westernised countries this decline is gradual and the signs become mostly noticeable after the 5 th decade of life and henceforth, where the individual slowly progresses to death over the next three to four decades. Given that the aging process is slow and gradual, it presents with opportunities and options that may ameliorate and improve the overall functional capacity of the organism. Small changes in function may be more amenable and likely to further slow down and possibly reverse some of the deleterious effects of aging, rather, than when the incremental changes are large. This overall effect may then translate into a significant compression of the deleterious aspects of human aging with a resultant increase in human life expectancy.
\end{abstract}

Keywords: human aging, life expectancy, lifespan, energy

\section{Introduction}

Understanding the difference between life expectancy and lifespan is the framework upon which any selective review of anti-aging interventions must originate from. Human life expectancy is determined from mortality data tables. Life expectancy is a statistical projection of the length a human being is expected to live based upon probabilities and assumptions of genetic predispositions, living conditions, medical discoveries and advances, natural disasters and other environmental factors. Morbidity statistics track presumed causes of death over time, producing trend data that can be factored into life expectancy tables (Mathers and Loncar 2005). Lifespan, however, is defined as the characteristic observed age of death for its very oldest individuals.

Throughout most of recorded human history, it has been recognized that poor socioeconomic and nutritional status, have been strongly associated with decreased life expectancy, a trend that is also very much evident today (US Gov. Report, 2005). Current data estimates, show that on average human life expectancy in western countries is approximately 82 years for women and 80 years for men (US Gov. Report, 2005).

In this brief selective review we present scientific evidence as to what anti aging practices may better serve to increase mean life expectancy and hence the human lifespan.

\section{Lifestyle}

Willet (2002) recently emphasized that genetic and environmental factors, including diet and lifestyle, both contribute to cardiovascular diseases, cancers, and other major 
causes of mortality and that numerous lines of evidence indicate that environmental factors are the most important in determining disease prevention. Hence, environmental factors may certainly have the strongest influence on life expectancy and hence lifespan (Figure 1) (Willett 2002; Vitetta et al 2005). Intertwined with nutritional practices and lifestyle is life stressor modification (Vitetta et al 2005).

Evidence continues to accumulate that strongly suggests that the state of a human being's mind - which associates psychosocial factors with emotional states such as depression and with behavioural dispositions that include hostility and psychosocial lifestyle stresses - can directly and significantly influence human physiologic function and, in turn, health outcomes.

Stressors and negative stress-related reactions have been documented and recognized to have multiple ill health related sequalae and exposure to chronic social stress has been associated with many systemic and mental disorders. These have significant deleterious outcomes that significantly decrease life expectancy (Ader 2003; Vitetta et al 2005).

Different research groups support the notion that health consequences are more likely to occur when unpredictable stressors of a social nature chronically induce physiological and behavioral adjustments that may create wear and tear on underlying physiological functions. When stressors challenge an organism's integrity, a set of physiological reactions is elicited to counteract the possible threat and adjust the physiological setting of the organism to the new situation. This has become known as the stress response (Ader 2003). Stresors and lifestyle choices can be a significant trigger for disease through immunomodulation of the immune system enhancing disease susceptibility, which can then affect life expectancy (Ader 2003). Immune function modulation influenced and expressed by stressful life experiences has a significant

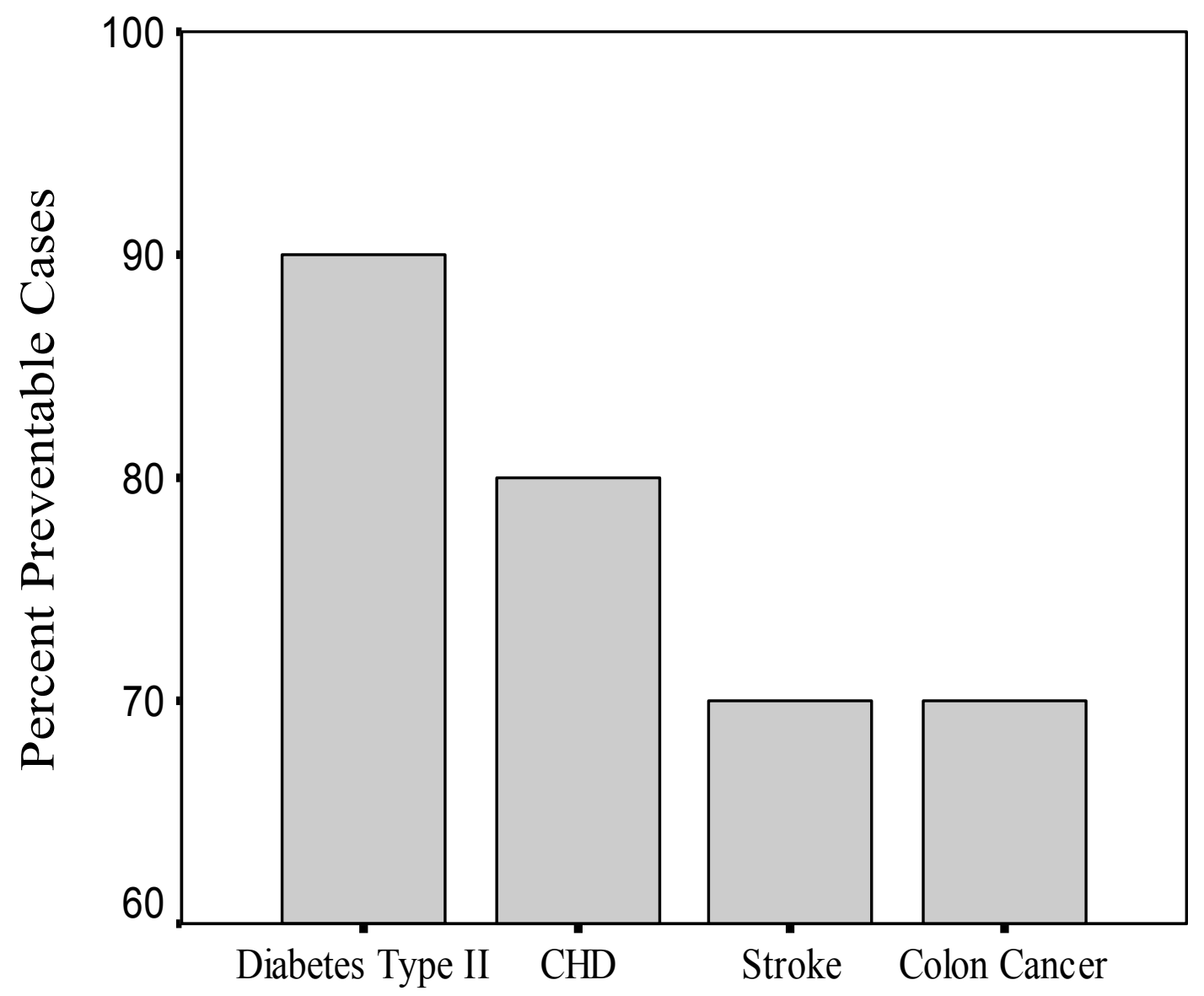

\section{Chronic Disease Type}

Figure I Preventable chronic diseases. Adapted and modified from Willett 2002. 
correlate with Willett's (2002) environmental trigger view of disease initiation.

\section{Nutrition}

Triggers for adverse and inappropriate nutritional practices in childhood have been shown to have psychosocial correlates in adult life with an increase in risk for disease (Jackson 2005). Obesity in humans, influenced by poor dietary choices and inactivity, are significantly associated with an increased risk of chronic diseases such as diabetes, high blood pressure, high cholesterol, cardiovascular diseases, asthma, arthritis, some cancers and overall poor health status, that can significantly decrease an individual's life expectancy.

$\mathrm{CR}$ is linked to nutritional choices. CR with nutritionally poor foods (eg, consuming half the quantity of a fast food) is still an unbalanced nutritional choice albeit in smaller portions and still provides no health advantage.

Recently it was reported and further confirmed, that a greater adherence to a traditional Mediterranean diet was associated with a significant reduction in total all cause mortality (Trichopoulou et al 2003). Meyer and colleagues (2006) have demonstrated that associating a CR diet with adherence to a Mediterranean type diet that consisted of whole grains, beans, fish, fruit, olive oil, and many different kinds of vegetables was beneficial for heart health. Hence when optimal nutritional choices are coupled to $\mathrm{CR}$, an increase in life expectancy is made possible. Such nutritional practices can serve to further expand the human life span.

\section{Caloric restriction (CR)}

Optimizing nutrition relates food intake to the benefits of prudent CR. That is, CR refers to a dietary regimen low in calories without under nutrition that leads to increased disease risk due to nutritional deficits. Following the pioneering work of McCay and Maynard (1935) over 70 years ago, CR was then first noted to significantly extend the life span of rodents. Since that time, the increase in longevity has been demonstrated to result from the limitation of total calories derived from carbohydrates, fats, or proteins to a level $25 \%-60 \%$ below that of control animals that were fed ad libitum (Sohal and Weindruch 1996; Weindruch and Sohal 1997; Weindruch et al 2001). The extension in lifespan can approach 50\% in rodents (Figure 2) (McCay et al 1935; Holloszt 1997). Moreover, CR has been shown to extend the lifespan in a broad range of organisms that include, yeast, rotifers, spiders, worms, fish, mice, and rats (Weindruch et al 2001). Emerging data show that its effect may also apply to non human primates (Lane et al 2001). Also
CR has been reported to delay a wide spectrum of diseases in different experimental animals such as kidney disease (Lane et al 2001), a variety of neoplasias (Fernandes et al 1976; Fernandes and Good 1984; Kubo et al 1984), autoimmune disease (Sarkar et al 1982; Engelman et al 1990) and diabetes (Shields et al 1991), and it reduces age associated neuronal loss in most mouse models of neurodegenerative disorders such as Parkinson's Disease (Duan and Mattson 1999) and Alzheimer's Disease (Zhu et al 1999). The CR regimen also prevents age-associated declines in psychomotor and spatial memory tasks (Ingram et al 1997) and loss of dendritic spines necessary for learning (Moroi-Fetters et al 1989) and improves the brain's plasticity and ability for self repair (Mattson 2000).

Numerous biomarkers of CR have been identified in rodents, such as temperature, and DHEAS, insulin and glucose levels (Roth et al 2002). Roth and colleagues (2002) have recently observed that body temperature and insulin and DHEAS levels were also altered in primates that had been subjected to $\mathrm{CR}$, hence validating the usefulness of these biomarkers in longer-lived species. More importantly, they have also shown that these parameters were altered in longer-lived men. Together these findings support the role these factors have as biomarkers of longevity in humans.

Recently, a trial on the effect of a 6 month CR diet on metabolic biomarkers such as energy expenditure, and oxidative stress in humans was completed (Heilbronn et al 2006). The report showed that prolonged CR significantly reduced two biomarkers of longevity, namely, fasting insulin level and body temperature. This is the first human study to show that $\mathrm{CR}$, in addition to significant reductions in well known biomarkers of aging, also caused a metabolic adaptation in CR individuals and a reduction in DNA fragmentation that reflected less DNA damage (Heilbronn et al 2006).

Important genetic links and regulators of organism life span have been uncovered in the form of sirtuins (Sauve et al 2006) Sirtuins are a family of $\mathrm{NAD}^{+}$- dependent protein deacetylases widely distributed in all phyla of life. In multicellular organisms, sirtuins deacetylate histones and transcription factors that regulate stress, metabolism, and survival pathways. Sir2 is the founding member of a large and diverse family of these protein-modifying sirtuin enzymes, which regulate key pathways throughout biology, in eubacteria, archaea, eukaryotes, and even viruses (Sauve et al 2006).

The link between CR and Sir2 and aging has been reviewed elsewhere (cf. for reviews: refer Sinclair and Guarente 1997; Guarente and Picard 2005; Suave et al 2006). We further point out that there is an extensive body of evidence that 


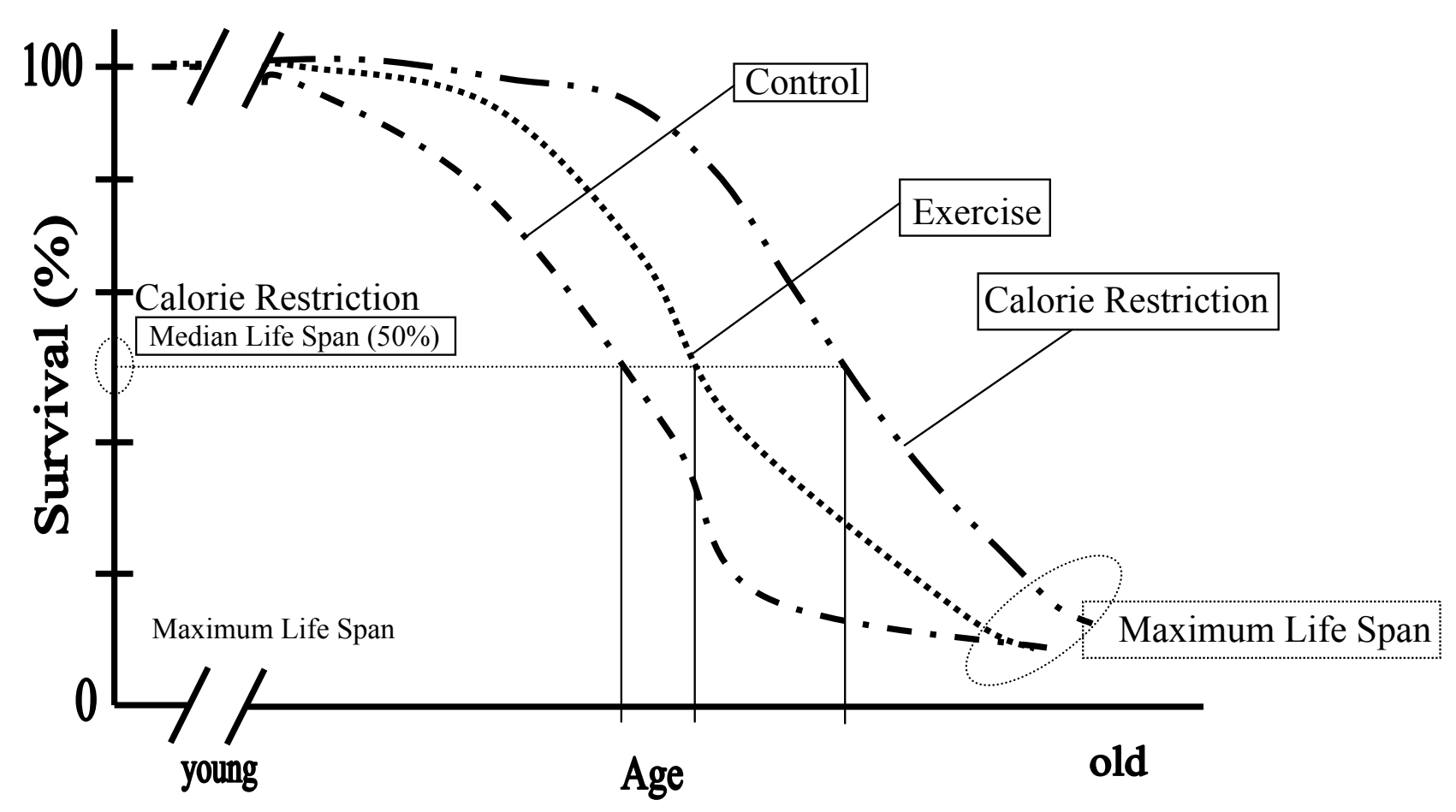

Figure 2 Effect of CR and Exercise on Survival in rats. Adapted and modified from Holloszy 1995.

suggests that sirtuins are involved in promoting longevity, particularly longevity associated with CR regimens, in several organisms. The sirtuin $-\mathrm{CR}$ relationship remains complex however. Recently Suave and colleagues (Sauve et al 2006) hypothesized that mammalian SIRT1 may mediate significant changes in tissues and endocrine systems through the sensing of low CR diets and triggering physiological changes that benefit mammals with health and hence longevity.

Plant factors such as resveratrol have been shown to increase longevity in several organisms by regulating Sir2/SIRT1 (cf. for review: Baur and Sinclair 2006). In mammals, there is growing evidence that resveratrol can prevent or delay the onset of cancer, heart disease, ischaemic and chemically induced injuries, diabetes, pathological inflammation and viral infection. These effects are observed despite extremely low bioavailability and rapid clearance of resveratrol from the circulation (Baur and Sinclair 2006). Resveratrol has been suggested to be associated with the prevention of age-related diseases such as cancer because of its regulation of transcription factors that control tumuor cell survival (Guarente and Kenyon 2000; Howitz et al 2003; Nemoto et al 2005; Storz 2005).

\section{Mitochondria and free radicals}

That mitochondria have a pivotal role in the effective provision of energy to eukaryotic cells is an undisputed scientific fact. Cellular mechanisms regulating energy utilization must function properly to sustain life. Adenosine 5 -triphosphate (ATP) is the ubiquitous energy storage molecule produced in several cellular processes. Aerobic energy metabolism requires a cellular investment, namely, molecular oxygen. Humans rely on mitochondria to synthesize and export ATP. The process is initiated by the transfer of electrons derived from food sources along a series of mitochondrial respiratory-chain carriers until they are consumed with the production of water from the utilization of oxygen. A single eukaryotic cell may contain between several hundred to thousands of these energy organelles. With increased aging, there is a decrease in mitochondrial energy output (Singh 2006). Hence in aerobic animals, mitochondrial health for effective energy provision is central to life.

The free radical theory of aging, as formulated by Denham Harman (1956), is supported by observations that the life span of most organisms is roughly proportional to their metabolic rate and thus due to the rate at which the organism generates mitochondria derived reactive oxygen species (ROS). This view however, is over simplistic and may require modification within the confines of aging. Cellular generated ROS contributing to the overall production of ROS are apparently traced back to the mitochondria (Lenaz et al 2002). ROS have been viewed as mostly 
deleterious to health and hence aging. Reports that show that in a wide spectrum of animal species, dietary antioxidants or caloric restriction, as well as chemical antioxidants or increased expression of antioxidant proteins, can lower mitochondria ROS production, which translates into an extension of the life span of these species which have served to support the free radical theory of aging (Orr and Sohal 1994; Sohal and Weindruck 1996; Parkes et al 1998; Sun and Tower 1999; Taub et al 1999; Schon 2000; Finkel and Holbrook 2000; Xu and Finkel 2002) However, it is known that ROS are generated in multiple cellular compartments and by multiple enzyme systems within the cell and have cellular signaling functions that are critical for the normal physiological function of the cell (Rhee et al 2003; Linnane et al 2007a, 2007b, 2007c).

Important contributions to the production of ROS within cells, include proteins within the plasma membrane, such as the growing family of NADPH oxidases; lipid metabolism within the peroxisomes; protein synthesis within the endoplasmic reticulum; as well as the activity of various cytosolic enzymes such as the cyclo-oxygenases (Linnane and Eastwood 2004; Moldovan and Moldovan 2004; Balaban et al 2005). The generation of mitochondrial ROS is a consequence of oxidative phosphorylation, a process that uses the controlled and regulated oxidation of $\mathrm{NADH}$ or FADH to generate a potential energy for protons across the mitochondrial inner membrane (ROS produced by mitochondria have been demonstrated to have important and specific roles in cellular signalling (Linnane and Eastwood 2004). The notion that the mitochondria are the sole most abundant site of ROS formation is currently an area of much discussion and debate (Rhee et al 2003; Balaban et al 2005; Linnane et al 2007b).

The disruption of mitochondrial functions has been implicated in more than 40 known diseases, including atherosclerosis, ischemic heart disease, cancer, diabetes, and neurodegenerative diseases such as Alzheimer's disease, Parkinson's disease, Huntington's disease, and amyotrophic lateral sclerosis (cf. for reviews: McKenie 2004; Wallace 2005). Together these data indicate that mitochondrial health is an important factor for health and aging. Current and future research would aim to further improve and preserve mitochondrial function. Although further research is warranted, recent reports show that supplementation with coenzyme $\mathrm{Q}_{10}$ shows promise in maintaining the health of mitochondria (Kagan et al 1999; Crestanello et al 2002; McKenzie et al 2004; Somayagulu et al 2005; Linnane et al 2007b).
Further, mimetics of specific cellular antioxidants such as the SOD/catalase mimetic EUK-134 can be used to assist in reducing oxygen radicals and are already in clinical trials (Melov et al 2000).

\section{Hormones}

In humans there is a progressive decrease in hormone synthesis as well as a loss of hormone receptors with age. The elderly have demonstrated significantly lower levels of production of most hormones compared to young adults (Hertogue 2005). There is a voluminous body of scientific literature concerned with hormonal deficits with aging. That is, levels of growth hormone $(\mathrm{GH})$ and insulin like growth factor-1 (IGF-1), melatonin (nocturnal), TSH, thyroid hormones (T3), calcitonin, DHEA (sulphated form in and its urinary 17-keto-metabolites), aldosterone, estrogens, testosterone in men and women have been demonstrated to progressively decrease with age in adults (for extensive review and individual references refer Hertogue 2005).

Hormonal deficits are not merely limited to menopause, andropause (male menopause), somatopause (adult growth hormone deficiency), and other sex hormone related diseases, they are now implicated in conditions such as obesity (Cranny et al 2006; Pasquali and Gambineri 2006), osteoporosis (Bennett 2005), fibromyalgia (Cleare 2004) chronic fatigue syndrome (Cleare 2004) cancer (Simonenko et al 2006) attention deficit problems (Cheng 2005) and possibly others as yet that remain to be elucidated.

Conventional medicine treatments have only severed to address hormone depletion, ignoring the mild and moderate deficiencies; whereas the anti-aging medicine model focuses on mild, moderate, and severe deficiencies. Symptoms and severity of symptoms will be proportional to the level of deficiency for each hormone (Anton et al 2005). Only recently has the science of endocrinology started focusing on advanced testing methods, taking into account reference ranges based on age as well as gender, free or bioavailable hormone levels versus bound hormone levels, and ratios between major antagonistic hormones to titrate patients and achieve an optimum balance. Titration and optimum balance have been used with thyroid and insulin hormones, and has not been universally applied to all other hormones, till recent times. Ongoing research in the field of longevity should include the use of nutrient and hormonal precursors to augment the body's ability to make more of its own hormonal compounds thus limiting structural and DNA damage in the latter decades of life. 


\section{Concluding remarks}

The purest use of the term "biological aging" confines it exclusively to molecular changes. Hayflick (2003) defines aging in biological systems as a stochastic process that occurs systemically after reproductive maturity in animals that reach a fixed size in adulthood and that it is then caused by the escalating loss of molecular fidelity that ultimately exceeds repair capacity. This then increases the vulnerability to pathology or age-associated diseases. Further, that there is a finitude associated with aging that eventually leads to death (Hayflick 2003). Grimley Evans (2000) defined senescent changes as de novo structural and functional alterations that are not part of the developmental program. In this selective and brief review we have summarized the scientific evidence that may prevent disease and maintain the molecular integrity of the organism, thus expanding the mean life expectancy whilst operating within the framework of normal physiological function and repair. We find that whilst aging is not irreversible and inevitable, certain key factors may add significant benefits to human mean life expectancy.

There is a vast array of scientific literature that concludes that lifestyle modification factors that optimize nutrition, physical activity and mental health have significant correlates with reducing the risks of disease in later life that is translates into an increased mean life expectancy. Further, although the correction of all hormonal deficits by an adequate multiple hormone replacement therapy, is not currently possible, hormone therapy does constitute a viable solution to prevent and treat most hormonal deficits due to pathological aging. Hertogue (2005) recently reported that even though we are not presently able to abrogate senescence by hormone treatments because of the unavailability as medications of all hormones that decline with age, the future appears promising.

As a final note; with the onset of the 21 st century, it is apparent that we stand on the horizon of a revolution in anti aging therapies and technologies. A promising therapeutic modality, the concept of stem cell treatments, will within the next decade, allow a markedly expanded quality of health and possibly also further increase the mean human life expectancy. Recent advances by major corporations, both private and public, have documented that we are already capable of taking stem cells from an individual and selectively copying these cells along with their DNA components. Thus making possible the restoration and rejuvenation properties that human bodies lose as they age. The prospects for use of human embryonic stem cells derivatives in regenerative medicine hence are significant (Trouson 2006). If, the potential contributions to human regenerative medicine can be realized, significant alterations and enhancement of mean life expectancy in humans will be possible.

\section{References}

Ader R. 2003. Conditioned immunomodulation: research needs and directions. Brain Behav Immun, (Suppl 1):S51-7. Review.

Anton B, Vitetta L, Cortizo F, Sali A. 2005. Can we delay aging? The biology and science of aging. Ann N Y Acad Sci, 1057:525-35.

Balaban RS, Nemoto S, Finkel T. 2005. Mitochondria, oxidants, and aging. Cell, 120:483-95.

Baur JA, Sinclair DA. 2006. Therapeutic potential of resveratrol: the in vivo evidence. Nat Rev Drug Discover, 5:493-506.

Bennett R. 2005. Growth hormone in musculoskeletal pain states. Curr Pain Headache Rep, 9:331-8.

Cheng SY. 2005. Isoform-dependent actions of thyroid hormone nuclear receptors: lessons from knockin mutant mice. Steroids, 70:450-4.

Cleare AJ. 2004. The HPA axis and the genesis of chronic fatigue syndrome. Trends Endocrinol Metab, 15:55-9.

Cranney A, Papaioannou A, Zytaruk N, et al. 2006. Parathyroid hormone for the treatment of osteoporosis: a systematic review. CMAJ, 175:52-9.

Crestanello JA, Doliba NM, Doliba NM, et al. 2002. Effect of coenzyme Q10 supplementation on mitochondrial function after myocardial ischemia reperfusion. J Surg Res, 102:221-8.

Duan W, Mattson MP. 1999. Dietary restriction and 2-deoxyglucose administration improve behavioral outcome and reduce degeneration of dopaminergic neurons in models of Parkinson's disease. J Neurosci Res, 57:195-206.

Engelman RW, Day NK, Chen RF, et al. 1990. Calorie consumption level influences development of $\mathrm{C} 3 \mathrm{H} / \mathrm{Ou}$ breast adenocarcinoma with indifference to calorie source. Proc Soc Exp Biol Med, 193:23-30.

Fernandes G, Yunis EJ, Good RA. 1976. Suppression of adenocarcinoma by the immunological consequences of calorie restriction. Nature, 263:504-7.

Fernandes G, Good RA. 1984. Inhibition by restricted calorie diet of lymphoproliferative disease and renal damage in MRL/lpr mice. Proc Natl Acad Sci, 81:6144-8.

Finkel T, Holbrook NJ. 2000. Oxidants, oxidative stress and the biology of aging. Nature, 408:239-47.

Global Population Aging in the 21 st Century and Its Economic Implications. 2005. URL: http://www.cbo.gov/ftpdocs/69xx/doc6952/12-12-Global. pdf.

Grimley Evans J. 2000. 21st Century: Review: ageing and medicine. J Intern Med, 247:159-67.

Guarente 1, Kenyon C. 2000. Genetic pathways that regulate aging in model organisms. Nature, 408:255-62.

Guarente L, Picard F. 2005. Cell, 120:473-82.

Harman D. 1956. Aging: a theory based on free radical and radiation chemistry. J Gerontol, 11:298-300.

Hayflick L. 2003. Living forever and dying in the attempt. Exp Gerontol, 38:1231-41.

Heilbronn LK, de Jonge L, Frisard MI, et al. 2006. Effect of 6-month calorie restriction on biomarkers of longevity, metabolic adaptation, and oxidative stress in overweight individuals: a randomized controlled trial. JAMA, 295:1539-48.

Hertogue T. 2005. The "multiple hormone deficiency" theory of aging: is human senescence caused mainly by multiple hormone deficiencies. Ann N Y Acad Sci, 1057:448-65. Review.

Holloszt JO. 1997. Mortality rate and longevity of food-restricted exercising male rats: a reevaluation. J Appl Physiol, 82:399-403.

Howitz KT, Bitterman KJ, Cohen HY, et al. 2003. Small molecule activators of sirtuins extend Saccharomyces cerevisiae lifespan. Nature, 425:191-6.

Ingram DK, Weindruch R, Spangler EL, et al. 1987. Dietary restriction benefits learning and motor performance of aged mice. J Gerontol, 42:78-81. 
Jackson AA. 2005. Integrating the ideas of life course across cellular, individual, and population levels in cancer causation. J Nutr, 135(12 Suppl):2927S-33S. Review.

Johnson PR, Stern JS, Horwitz BA, et al. 1997. Longevity in obese and lean male and female rats of the Zucker strain: Prevention of hyperphagia. Am J Clin Nutr, 66:890-903.

Kagan T, Davis C, Lin L, et al. 1999. Coenzyme Q10 can in some circumstances block apoptosis, and this effect is mediated through mitochondria. Ann NY Acad Sci, 887:31-47.

Kubo C, Day NK, Good RA. 1984. Influence of early or late dietary restriction on life span and immunological parameters in MRL/Mp-lpr/lpr mice. Proc Natl Acad Sci, 81:5831-5.

Lane MA, Black A, Handy A, et al. 2001. Caloric restriction in primates. Ann NY Acad Sci, 928:287-95.

Lenaz G, Bovina C, D'Aurelio M, et al. 2002. Role of mitochondria in oxidative stress and aging. Ann N Y Acad Sci, 959:199-213. Review.

Linnane AW, Eastwood H. 2004. Cellular redox poise modulation; the role of coenzyme Q10, gene and metabolic regulation. Mitochondrion, 4:779-89.

Linnane AW, Kios M, Vitetta L. 2007a. The essential roles of superoxide radical and nitric oxide formation for healthy aging. Mitochondrion, $7: 1-5$.

Linnane AW, Kios M, Vitetta L. 2007b. Healthy aging: regulation of the metabolome by cellular redox modulation and prooxidant signaling systems. The essential roles of superoxide anion and nitric oxide. Experimental Gerontology, 8:445-67.

Linnane AW, Kios M, Vitetta L. 2007c. Coenzyme Q(10) - its role as a prooxidant in the formation of superoxide anion/hydrogen peroxide and the regulation of the metabolome. Mitochondrion, (Suppl 1):S51-61.

Mathers CD, Loncar D. 2005. Updated projections of global mortality and burden of disease, 2002-2030: data sources, methods and results World Health Organization -http://www.who.int/healthinfo/statistics/ bodprojectionspaper.pdf.

Mattson MP. 2000. Neuroprotective signaling and the aging brain: Take away my food and let me run. Brain Res, 886:47-53.

McCay CM, Cromwell MF, Maynard LA. 1935. The effect of retarded growth upon the length of life span and upon the ultimate body size. J Nutr, 10:63-79.

McKenzie M, Liolitsa D, Hanna MG. 2004. Mitochondrial disease: mutations and mechanisms. Neurochem Res, 3:589-600. Review.

Melov S, Ravenscroft J, Malik S, et al. 2000. Extension of life-span with superoxide dismutase/catalase mimetics. Science, 289:1567-9.

Meyer TE, Kovacs SJ, Ehsani AA, et al. 2006. Long-Term Caloric Restriction Ameliorates the Decline in Diastolic Function in Humans. J Amer Coll Cardiol, 47:398-402.

Moldovan L, Moldovan NI. 2004. Oxygen free radicals and redox biology of organelles. Histochem Cell Biol, 122:395-412.

Moroi-Fetters SE, Mervis RF, London, et al. 1989. Dietary restriction suppresses age-related changes in dendritic spines. Neurobiol Aging, 10:317-22.

Nemoto S, Fergusson MM, Finkel T. 2005. SIRT1 functionally interacts with the metabolic regulator and transcriptional coactivator PGC- $1 \alpha$. $J$ Biol Chem, 280:16456-60.

Orr WC, Sohal RS. 1994. Extension of life-span by overexpression of superoxide dismutase and catalase in Drosophila melanogaster. Science, 263:1128-30.

Parkes TL, Elia AJ, Dickinson D. 1998. Extension of Drosophila lifespan by overexpression of human SOD1 in motorneurons. Nat Genet, 19:171-4.

Pasquali R, Gambineri A. 2006. Metabolic effects of obesity on reproduction. Reprod Biomed Online, 12:542-51.
Rhee CG, Chang TS, Bae YS, et al. 2003. Cellular regulation by hydrogen peroxide. J Am Soc Nephrol, 14(8 Suppl 3):S211-5. Review.

Roth GS, Lane MA, Ingram DK, et al. 2002. Biomarkers of caloric restriction may predict longevity in humans. Science, 297:811.

Sarkar NH, Fernandes G, Telang NT, et al. 1982. Low-calorie diet prevents the development of mammary tumors in $\mathrm{C} 3 \mathrm{H}$ mice and reduces circulating prolactin level, murine mammary tumor virus expression, and proliferation of mammary alveolar cells. Proc Natl Acad Sci, 79:7758-62.

Sauve AA, Wolberger C, Schramm VL, et al. 2006. The Biochemistry of Sirtuins. Annu Rev Biochem, 75:435-65.

Schon EA. 2000. Mitochondrial genetics and disease. Trends Biochem Sci, 25:555-60.

Shields BA, Engelman RW, et al. 1991. Calorie restriction suppresses subgenomic mink cytopathic focus-forming murine leukemia virus transcription and frequency of genomic expression while impairing lymphoma formation. Proc Natl Acad Sci, 88:11138-42.

Simonenko VB, Dulin PA, Makanin MA. 2006. Somatostatin analogues in treatment of gastrointestinal and pancreatic neuroendocrine tumors. Klin Med (Mosk), 84:4-8.

Sinclair DA, Guarente L. 1997. Cell, 91:1033-42.

Singh KK. 2006. Mitochondria damage checkpoint, aging, and cancer. Ann N Y Acad Sci, 1067:182-90. Review.

Sohal RS, Weindruch R. 1996. Oxidative stress, caloric restriction, and aging. Science, 273:59-63.

Somayajulu M, McCarthy S, Hung M, et al. 2005. Role of mitochondria in neuronal cell death induced by oxidative stress; neuroprotection by Coenzyme Q10. Neurobiol Dis, 18:618-27.

Storz P. 2005. Reactive oxygen species in tumor progression. Front Biosci, 10:1881-96.

Sun J, Tower J. 1999. FLP recombinase-mediated induction of $\mathrm{Cu} / \mathrm{Zn}$ superoxide dismutase transgene expression can extend the life span of adult Drosophila melanogaster flies. Mol Cell Biol, 19:216-28.

Taub J, Lau JF, Ma C, et al. 1999. A cytosolic catalase is needed to extend adult lifespan in C. elegans daf-C and clk-1 mutants. Nature, 399:162-6.

Trichopoulou A, Costacou T, Bamia C, et al. 2003. Adherence to a Mediterranean diet and survival in a Greek population. $N$ Engl J Med, 348:2599-608.

Trouson A. 2006. The production and directed differentiation of human embryonic stem cells. Endocr Rev, 27:208-19.

Vitetta L, Anton A, Cortizo F, et al. 2005. Mind-body medicine: stress and its impact on overall health and longevity. Ann NY Acad Sci, 1057:492-505.

Wallace DC. 2005. A mitochondrial paradigm of metabolic and degenerative diseases, aging, and cancer: a dawn for evolutionary medicine. Annu Rev Genet, 39:359-407. Review.

Weindruch R, Keenan KP, Carney JM, et al. 2001. Caloric restriction mimetics: Metabolic interventions. J Gerontol A Biol Sci Med Sci, 56 Spec No 1:20-33.

Weindruch R, Sohal RS. 1997. Seminars in medicine of the Beth Israel Deaconess Medical Center. Caloric intake and aging. NEJM, 337:986-94.

Willett W. 2002. Balancing life-style and genomics research for disease prevention. Science, 296:695-8.

$\mathrm{Xu}$ D, Finkel T. 2002. A role for mitochondria as potential regulators of cellular life span. Biochem Biophys Res Commun, 294:245-8.

Zhu H, Guo Q, Mattson MP. 1999. Dietary restriction protects hippocampal neurons against the death-promoting action of presenilin-1 mutation. Brain Res, 842:224-9. 
